\section{Prevalência e fatores associados à prematuridade entre gestantes submetidas à inibição de trabalho de parto prematuro}

\section{Prevalence and risk factors associated to preterm delivery among pregnant women submitted to preterm labor inhibition treatment}

Lucila Coca Bezerra 1

Sonia M. Junqueira V. de Oliveira 2

Maria do Rosário Dias de Oliveira Latorre 3

\begin{abstract}
Objectives: to identify preterm delivery prevalence in pregnant women submitted to preterm delivery inhibition treatment and to analyze associated factors.

Methods: cross sectional study with data collected from 163 pregnant women medical files seen from 1995-2000 at the University Hospital of the University of São Paulo, Brazil. The dependent variable was preterm delivery and the independent ones were: age, education, job, parity, companion, smoking, prior urinary infection, number of prenatal medical visits and birth interval. Analysis was performed through association by the Chi-square test and univariate and multiple logistic regression models.

Results: $66.3 \%$ of the women had preterm deliveries and in $22.7 \%$ of the cases, delivery occurred before 34 weeks. There was a statistically significant association between preterm birth and no partner, nulliparity and a low number of prenatal visits.

Conclusions: special attention should be given to nulliparous pregnant women with reduced numbers of prenatal medical visits submitted to preterm delivery inhibition treatment with the objective of preventing premature births.
\end{abstract}

Key words Infant, premature, Labor obstetric, Multivariate analysis

\section{Resumo}

Objetivos: identificar a prevalência de parto prematuro em gestantes submetidas ao tratamento de inibição de trabalho de parto prematuro e analisar os fatores associados.

Métodos: estudo transversal, com dados coletados de 163 prontuários de gestantes submetidas a tratamento de inibição de trabalho de parto prematuro atendidas em 1995-2000, no Hospital Universitário da Universidade de São Paulo, Brasil. A variável dependente constituiu-se na ocorrência de parto prematuro e as independentes foram: idade, escolaridade, ocupação, paridade, companheiro, tabagismo, infecção urinária prévia, número de consultas pré-natal e intervalo interpartal. A análise foi feita pelo teste de associação pelo Qui-quadrado e modelos de regressão logística univariado e múltiplo.

Resultados: $66,3 \%$ das gestantes tiveram filhos prematuros e, em $22,7 \%$ dos casos, o parto ocorreu antes de 34 semanas. Houve associação estatística significativa entre parto prematuro e ser nulípara e apresentar número baixo de consultas pré-natal.

Conclusões: atenção especial deve ser dada às gestantes nulíparas e com número reduzido de consulta pré-natal submetidas ao tratamento de inibição de trabalho de parto prematuro, com a finalidade de prevenir esse evento.

Palavras-chave Prematuro, Trabalho de parto, Análise múltivariada 


\section{Introdução}

Apesar dos avanços tecnológico e científico, a prematuridade, ainda hoje, é um grande problema na Obstetrícia e na Neonatologia, constituindo-se em uma das causas de morbidade e mortalidade neonatal. Dados de 2003 do Ministério da Saúde apontam prevalência de recém-nascidos (RN) prematuros em nosso país de $6,4 \%$, com alguma variação dependendo da região. ${ }^{1}$ Um estudo realizado em Campinas com 580 gestantes de baixo risco constatou $5,5 \%$ de parto prematuro. ${ }^{2}$

O estilo de vida da gestante, como o hábito de fumar, a desnutrição, o ganho ponderal inadequado na gestação e o estresse psicológico materno são algumas causas de parto prematuro. ${ }^{3}$ Além disso, também estão relacionados baixo nível socioeconômico, mulheres jovens, sem companheiro, e fatores médicos, como prematuridade anterior e sangramento vaginal persistente no início da gestação. 4

A avaliação precisa do início do trabalho de parto prematuro (TPP) é difícil, pois essa patologia tem etiologia multifatorial, sendo o diagnóstico realizado com base na dilatação cervical progressiva e, nesses casos, é muito tarde para tentar iniciar o tratamento preventivo. ${ }^{4}$ Muitas vezes, o TPP progressivo cessa antes mesmo de se iniciar o tratamento. $\mathrm{O}$ fato de inibir as contrações uterinas não significa que o parto será postergado por um período que seria clinicamente benéfico. Mesmo que a gestação seja prolongada por um período, isso não significa que o resultado será melhor. Toda droga ou tratamento suficientemente eficaz para inibir as contrações uterinas pode ter outros efeitos na mãe e no concepto que são indesejáveis e devem ser levados em consideração. 5

A inibição das contrações deve ser o último recurso para impedir o trabalho de parto prematuro aplicada aproximadamente em $20 \%$ dos casos, em razão da fase adiantada em que normalmente está o trabalho de parto ou devido a freqüentes associações com patologias maternas ou fetais. ${ }^{6}$ São consideradas contra-indicações absolutas para a tocólise: o sofrimento fetal, anomalia incompatível com a vida, corioamnionite, eclâmpsia ou pré-eclâmpsia severa, hemorragia materna de qualquer etiologia e intenso comprometimento do desenvolvimento fetal. ${ }^{3}$ As contra-indicações relativas são: hipertensão arterial, nefropatia crônica, diabetes de difícil controle, restrição do crescimento fetal, poli-hidrâmnio, trabalho de parto com mais de quatro centímetros de dilatação cervical e maturidade pulmonar comprovada em gestações com mais de 34 semanas. 3

O diagnóstico precoce, sobretudo a percepção da ocorrência das contrações uterinas, é fundamental para detecção precoce do TPP.7 Muitos critérios têm sido empregados para descrever o êxito do tratamento. Os mais usados relatam o tempo de inibição das contrações, o número de horas ou dias que retardou o nascimento, o número de partos com 36 ou 37 semanas de gestação. 8 É considerado um sucesso quando o parto é postergado por 24 ou 48 horas, para introdução da corticoterapia ou transferência in útero para um hospital com mais recursos neonatais. 8

A proposta deste estudo foi identificar a prevalência de parto prematuro em gestantes que fizeram tratamento para inibir o TPP e analisar os fatores associados ao nascimento prematuro.

\section{Métodos}

Trata-se de um estudo transversal com coleta de dados de prontuários de gestantes atendidas no Centro Obstétrico do Hospital Universitário (HU) da Universidade de São Paulo (USP). Participaram do estudo todas as 163 mulheres atendidas no HU/USP, com diagnóstico de TPP, sem nenhuma contra-indicação para tratamento de inibição, tendo tido seus partos de janeiro de 1995 a agosto de 2000.

No período citado, o protocolo estabelecido pela Clínica Obstétrica do HU/USP para tratamento medicamentoso do trabalho de parto prematuro consistia de repouso absoluto e hidratação endovenosa. Após uma hora, caso as contrações uterinas persistissem, eram introduzidas cinco ampolas de isoxsuprina diluídas em $500 \mathrm{ml}$ de soro glicosado a $5 \%$, começando com 4 gotas $/ \mathrm{min}$ (50 $\mathrm{mcg} / \mathrm{min})$ e aumentando 4 gotas/min a cada 20 minutos (máximo até 40 gotas/min). Quando obtida a dose mínima necessária para inibir as contrações uterinas, a mulher era mantida por duas horas, com redução gradativa até atingir a dose inicial. No caso de contrações ausentes, durante duas horas, a medicação endovenosa era substituída por um comprimido via oral de 10 miligramas a cada seis horas. A gestante era encaminhada ao alojamento-conjunto, permanecendo internada por no mínimo 48 horas. ${ }^{9}$ Na mesma Instituição, Spallicci et al. ${ }^{9}$ usando igual protocolo de tratamento para inibir o TPP em 41 gestantes internadas, constataram que a inibição foi efetiva em $90,2 \%$ dos casos, com melhores resultados obtidos entre 12 e 24 horas após o início do uso da isoxsuprina.

Merece comentar que os betamiméticos (Salbutamol, Terbutalina, Ritodrina, Isoxsuprina, Orciprenalina e Hexoprenalina) são os fármacos mais utilizados nos últimos 20 anos para inibir as contrações 
uterinas. No entanto, deve-se salientar que a revisão de Anotayanonth et al. ${ }^{10}$ publicada pela Cochrane Library constatou que o uso do betamimético adiou o parto por 48 horas, mas não reduziu o número de nascimentos por um período maior de sete dias, concluindo que não existem dados suficientes para recomendar sua utilização. Da mesma forma, em relação à hidratação endovenosa ou oral na inibição do TPP, a revisão sistemática de Stan et al.11 concluiu pela inexistência de resultados eficazes no tratamento dessa intercorrência.

No atual estudo, a variável dependente foi parto prematuro e as variáveis independentes analisadas foram: número de internações, situação conjugal, idade, escolaridade, hábito de fumar, antecedente de parto prematuro, ocorrência de infecção do trato urinário, número de consultas de pré-natal, paridade e intervalo interpartal.

Por meio do software Epi-info 6, as freqüências absolutas e relativas das variáveis categóricas e as médias e medianas das variáveis quantitativas foram calculadas. A seguir, foram analisados os fatores associados pelo teste de associação pelo Quiquadrado com correção de Yates e análise conjunta dos mesmos por intermédio do modelo de regressão logística múltipla. Na análise múltipla, foram selecionadas todas as variáveis significativas no teste de associação pelo Qui-quadrado, sendo a medida de risco utilizada a odds ratio (OR) e seu respectivo intervalo de $95,0 \%$ de confiança. O nível de significância igual a 5,0 \% foi usado em todas análises.

\section{Resultados}

Do total de 163 gestantes com diagnóstico de TPP, sem contra-indicação para o tratamento de inibição, verificou-se que $66,3 \%$ dos partos ocorreram prematuramente. Dos 108 partos prematuros, 76 $(69,1 \%)$ foram por via vaginal e $34(30,9 \%)$ via abdominal. As indicações de cesárea para os partos prematuros foram: apresentação pélvica (nove casos), sofrimento fetal agudo (oito casos), iterativa (sete casos), gravidez gemelar (seis casos), descolamento prematuro de placenta (dois casos), vício pélvico (um caso) e distocia funcional (um caso). Dos 55 partos a termo, $41(74,5 \%)$ tiveram parto vaginal e $14(25,5 \%)$ cesárea. As indicações de cesáreas dos partos de termo foram: iterativa (três casos), sofrimento fetal agudo (três casos), distocia funcional (três casos), apresentação pélvica em gemelar (três casos) e iminência de eclampsia (um caso).

Os resultados apontaram que, do total das mulhe-
Tabela 1

Distribuição das gestantes, segundo a idade gestacional em semanas por ocasião do parto. Hospital Universitário da Universidade de São Paulo, São Paulo, janeiro de 1995 a agosto de 2000 .

\begin{tabular}{crr}
\hline $\begin{array}{c}\text { Idade gestacional } \\
\text { (em semanas) }\end{array}$ & $\mathbf{N}$ & $\%$ \\
\hline 29 & 8 & 4,9 \\
30 & 2 & 1,2 \\
31 & 7 & 4,3 \\
32 & 8 & 4,9 \\
33 & 12 & 7,4 \\
34 & 13 & 8,0 \\
35 & 20 & 12,3 \\
36 & 38 & 23,3 \\
$\geq 37$ & 55 & 33,7 \\
Total & 163 & 100,0 \\
\hline
\end{tabular}

res, $74,2 \%$ tiveram uma única internação, com duração média de cinco dias. Levando em conta a idade gestacional, constatou-se que, das 55 mulheres com partos a termo, $45(81,8 \%)$ tiveram uma internação, $9(16,4 \%)$ duas internações e somente uma mulher $(1,8 \%)$ três internações; ao passo que das 108 parturientes com parto pré-termo, $76(70,4 \%)$ apresentaram uma internação, 29 (26,8\%) duas internações e $3(2,8 \%)$ tiveram três internações. Após o tratamento de inibição, o parto foi postergado por 1 a 87 dias (média de 17,4 dias e mediana de 11 dias), correspondendo ao período de internação hospitalar e ao tratamento domiciliar.

A idade variou de 13 a 39 anos (média de 23,7 anos), e a maioria vivia com companheiro $(76,5 \%)$, tinha ensino fundamental $(61,3 \%)$, sem o hábito de fumar $(81,5 \%)$, sem antecedente de parto prematuro $(80,4 \%)$, não apresentava infecção urinária $(62,9 \%)$, era nulípara $(52,8 \%)$, fez quatro ou mais consultas de pré-natal $(80,0 \%)$ e apresentava intervalo interpartal acima de dois anos $(64,9 \%)$.

A maioria das gestantes $(69,3 \%)$ que foi submetida ao tratamento de inibição do TPP teve seus filhos com 35 ou mais semanas de gestação, sendo que $55(33,7 \%)$ dos recém-nascidos nasceram a termo, isto é, acima de 37 semanas. Convém destacar que, mesmo após o tratamento de inibição de TPP, 66,3\% ainda resultaram em partos prematuros (Tabela 1).

$\mathrm{Na}$ análise bivariada, os fatores de risco que não apresentaram significância estatística foram: idade, escolaridade, hábito de fumar, antecedente de parto prematuro, ocorrência de infecção urinária e intervalo interpartal (Tabela 2). 
Distribuição das características sociodemográficas e clínicas das parturientes segundo a ocorrência de prematuridade. Hospital Universitário da Universidade de São Paulo, São Paulo, janeiro de 1995 a agosto de 2000.

\begin{tabular}{|c|c|c|c|c|c|c|}
\hline \multirow[t]{2}{*}{ Características } & \multicolumn{2}{|c|}{ Parto prematuro } & \multicolumn{2}{|c|}{ Parto a termo } & \multirow[b]{2}{*}{ OR } & \multirow[b]{2}{*}{ IC95\% } \\
\hline & $\mathrm{n}$ & $\%$ & $\mathrm{n}$ & $\%$ & & \\
\hline \multicolumn{7}{|c|}{ Companheiro $(n=162)$} \\
\hline $\operatorname{sim}$ & 77 & 71,3 & 47 & 87,0 & 1 & \\
\hline Não & 31 & 28,7 & 7 & 13,0 & 2,70 & $1,03-7,35$ \\
\hline \multicolumn{7}{|l|}{ Idade $(n=163)$} \\
\hline$\leq 19$ & 33 & 30,6 & 13 & 23,6 & 1,42 & $0,64-3,21$ \\
\hline 19 & 75 & 69,4 & 42 & 76,4 & 1 & \\
\hline \multicolumn{7}{|c|}{ Escolaridade $(n=160)$} \\
\hline$\leq 8$ & 64 & 60,9 & 34 & 61,8 & 1,05 & $0,28-3,79$ \\
\hline $9+12$ & 32 & 30,5 & 16 & 29,1 & 1,11 & $0,27-4,50$ \\
\hline$\geq 12$ & 9 & 8,6 & 5 & 9,1 & 1 & \\
\hline \multicolumn{7}{|c|}{ Hábito de fumar ( $n=162)$} \\
\hline Não & 86 & 80,4 & 46 & 83,6 & 1 & \\
\hline Sim & 21 & 19,6 & 9 & 16,4 & 1,25 & $0,49-3,22$ \\
\hline \multicolumn{7}{|c|}{ Antecedente de prematuro $(n=163)$} \\
\hline Não & 88 & 81,5 & 43 & 78,2 & 1 & \\
\hline Sim & 20 & 18,5 & 12 & 21,8 & 0,81 & $0,34-1,96$ \\
\hline \multicolumn{7}{|c|}{ Infecção do trato urinário $(n=159)$} \\
\hline Não & 64 & 61,5 & 36 & 65,5 & 1 & \\
\hline Sim & 40 & 38,5 & 19 & 34,5 & 1,18 & $0,57-2,48$ \\
\hline \multicolumn{7}{|c|}{ Consultas $(n=156)$} \\
\hline$\leq 3$ & 26 & 25,7 & 5 & 9,1 & 5,62 & $1,69-19,79$ \\
\hline 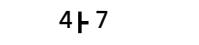 & 50 & 49,5 & 23 & 41,8 & 2,35 & $1,06-5,24$ \\
\hline$\geq 7$ & 25 & 24,8 & 27 & 49,1 & 1 & \\
\hline \multicolumn{7}{|c|}{ Paridade $(n=163)$} \\
\hline Nulípara & 65 & 60,2 & 21 & 38,2 & 3,82 & $1,59-9,31$ \\
\hline Primípara & 17 & 15,7 & 21 & 38,2 & 1 & \\
\hline Multípara & 26 & 24,1 & 13 & 23,6 & 2,47 & $0,89-6,93$ \\
\hline \multicolumn{7}{|c|}{ Intervalo interpartal $(n=73)$} \\
\hline$\leq 2$ anos & 15 & 38,5 & 8 & 23,5 & 2,03 & $0,66-6,41$ \\
\hline$\geq 3$ anos & 24 & 61,5 & 26 & 76,5 & 1 & \\
\hline
\end{tabular}

As variáveis associadas estatisticamente com o parto prematuro em gestantes submetidas à inibição foram: a falta de companheiro $(\mathrm{OR}=2,70)$, ser nulípara $(\mathrm{OR}=3,82)$ quando comparada com a primípara e o número de consultas pré-natal abaixo de 7 [(4 6 consultas) $\mathrm{OR}=2,35$ e $(<4$ consultas $) \mathrm{OR}=5,62]$
(Tabela 2).

A Tabela 3 apresenta os resultados do modelo final de regressão logística múltipla. Nesta análise, permaneceram como fatores associados, independentes da presença de companheiro, o fato de ser nulípara $(\mathrm{ORaj}=3,82)$ quando comparada com a 
Tabela 3

Análise múltipla final dos fatores associados ao parto prematuro em gestante submetidas à inibição do trabalho de parto prematuro. Hospital Universitário da Universidade de São Paulo, São Paulo, janeiro de 1995 a agosto de 2000 .

\begin{tabular}{|c|c|c|}
\hline Variável & $\mathrm{OR}_{\mathrm{aj}}$ & IC95\% \\
\hline \multicolumn{3}{|l|}{ Companheiro } \\
\hline Com & 1,00 & \\
\hline Sem & 1,81 & $0,68-4,82$ \\
\hline \multicolumn{3}{|l|}{ Paridade } \\
\hline Nulípara & 2,92 & $1,24-6.87$ \\
\hline Primípara & 1,00 & \\
\hline Multípara & 2,44 & $0,93-6,39$ \\
\hline \multicolumn{3}{|l|}{ Pré-natal } \\
\hline$\leq 3$ consultas & 4,26 & $1,36-13,73$ \\
\hline $4+7$ consultas & 2,12 & $0,99-4,56$ \\
\hline$\geq 7$ consultas & 1,00 & \\
\hline
\end{tabular}

primípara e o número de consultas de pré-natal abaixo de quatro $(\mathrm{ORaj}=4,26)$ quando comparada com ter "feito sete ou mais consultas". A existência de companheiro perdeu sua significância estatística por estar associada à paridade.

\section{Discussão}

A importância clínica do diagnóstico precoce do trabalho de parto pré-termo está na possibilidade de aumentar a duração da gestação, mediante intervenção em tempo hábil que permita melhorar o peso fetal ao nascer, associado à diminuição da morbidade e mortalidade neonatal. ${ }^{7}$

No atual estudo, a idade gestacional das gestantes para tratamento do TPP na admissão hospitalar variou de 25 a 36 semanas; dessas 26,9\% estavam com menos de 32 semanas. Apesar do tratamento de inibição do TPP, constatou-se que 66,3\%, dos nascimentos, ainda, ocorreram prematuramente. Em média, o parto foi postergado em 17,4 dias, o que é de suma importância para o desenvolvimento dessas crianças, pois 2,5 semanas a mais na idade gestacional podem mudar o prognóstico do recémnascido prematuro.

De acordo com os dados do estudo colaborativo sobre tratamento com esteróides durante o pré-natal, em $87,3 \%$ dos recém-nascidos a idade gestacional foi maior ou igual a 34 semanas, a grande maioria dos casos graves de mortalidade e morbidade decorrentes do parto pré-termo ocorreu antes da $34^{\mathrm{a}}$ semana de gravidez. ${ }^{12}$ Um estudo mais recente relata que os partos ocorridos entre 34 a 35 semanas tiveram mais complicações respiratórias, sepsis e maior tempo de hospitalização do que os com 35 a 36 semanas, sugerindo, assim, considerar a inibição naquele período. 13

Os resultados deste estudo apontam que a maioria das gestantes $(74,2 \%)$ teve somente uma internação para tratamento de TPP. Como era esperado, entre as mulheres com parto a termo, constatou-se menor frequiência de duas e três internações $(16,4 \%$ e $1,8 \%)$ comparadas com aquelas de parto pré-termo $(26,8 \%$ e $2,8 \%)$, respectivamente. Nesse sentido, é importante salientar que na orientação para a alta hospitalar devem ser abordados que o padrão contrátil está aumentado e, geralmente, as contrações uterinas são dolorosas, para a gestante e sua família identificarem o momento correto de voltar ao hospital para tratamento.

Foi constatado que, apesar do tratamento de inibição do TPP, a gestante sem companheiro apresentou maior risco de ter parto prematuro na análise univariada, comparada àquela com companheiro. No entanto, na análise múltipla, a variável companheiro perdeu sua significância estatística. Por outro lado, no estudo de Almeida et al.14 foi observado um número significativamente menor de parto prematuro nas mulheres que referiram relação do tipo marital, embora esses autores não tenham utilizado a análise múltipla das variáveis significativas. Outra pesquisa realizada em dois locais distintos, após ter feito análise ajustada para a paridade, encontrou associação estatística entre parto prematuro e mulher sem companheiro, em uma sociedade em que esse comportamento era pouco freqüente, porém não observou associação estatística, onde o comportamento de mães solteiras era mais comum. ${ }^{15}$ Assim, é importante considerar o contexto social em que a mulher vive.

Nesse sentido, em nossa sociedade, o estilo de vida da mulher sem companheiro parece contribuir para aumentar o estresse psicológico. Ainda hoje, existem famílias, sobretudo de baixa renda, que expulsam de casa a filha solteira ao tomar conhecimento de sua gravidez, e na cidade de São Paulo, os locais solidários a essas gestantes encontram-se sempre lotados. 16

Os dados demonstraram que a idade materna não apresentou associação com nascimento prematuro. Resultado semelhante foi verificado comparando gestantes de até 19 anos com aquelas acima de 20 anos. 17 Outra pesquisa, realizada em São Luís do 
Maranhão, observou significância estatística entre prematuridade e idade materna menor que 18 anos. ${ }^{18}$

Embora a baixa escolaridade esteja associada ao parto prematuro, no estudo de Robaina Castellano et al., 19 essa variável não foi significativa na atual investigação. A justificativa pode ser o fato da gestante ter acesso ao serviço de saúde para tratamento. Silva et al.20 encontraram associação do tabagismo com parto prematuro; no entanto, no presente estudo, o hábito de fumar não teve significância estatística, pois foi observado grande número de não fumantes tanto entre as mulheres com parto prematuro $(80,4 \%)$ como naquelas com parto a termo $(83,6 \%)$.

A infecção do trato urinário está associada com o parto pré-termo, 21 porém, neste estudo, todas as gestantes internadas com TPP realizaram exame de urina para diagnóstico de ITU e, na ocorrência de infecção, todas as mulheres foram tratadas, assim, essa variável não foi significativa no sucesso ou não do tratamento de inibição. Além disso, ter antecedente de parto prematuro também aumenta significativamente a chance de uma nova recorrência. 14 No entanto, na atual investigação e em outro estudo, 9 realizados no mesmo hospital, não foram encontradas associação estatística com antecedente de parto prematuro.

A associação entre prematuridade e a falta de assistência pré-natal é documentada por diversos estudos. 18 Neste trabalho os achados apontam associação significativa inversamente proporcional entre o número de consultas e o parto prematuro. Outras pesquisas, também, observaram maior número de recém-nascidos pré-termo em gestantes com cinco ou menos consultas de pré-natal, 21 quando a mulher não fez nenhuma consulta de prénatal, 63,6\% tiveram filhos a termo e naquelas em que o número de consultas foi igual ou maior que seis, os recém-nascidos a termo totalizaram $90 \% .22$

Quando se compara uma mulher com parto prematuro com outra a termo, constata-se que a segunda teve maior possibilidade de ter ido a mais consultas, visto que o parto ocorreu com maior idade gestacional. Por outro lado, se considerarmos a recomendação do Ministério da $\operatorname{Saúde}^{23}$ de que a gestante deve iniciar o acompanhamento pré-natal com pelo menos 12 semanas e o retorno a cada quatro semanas, a gestante sem nenhuma intercorrência estaria com, pelo menos, cinco consultas ao completar 29 semanas, que seria o número mínimo de consultas esperado. Tendo em vista essa consideração, merece reforçar a importância da assistência pré-natal para identificar a gestante com maior possibilidade de parto prematuro para seguimento e orientação quanto aos sinais de TPP para a prevenção desta complicação.

A distribuição das mulheres, segundo a paridade, foi $52,8 \%$ de nulíparas, $23,3 \%$ de primíparas e $23,9 \%$ de multíparas. Igualmente, no estudo de Spallicci et al. ${ }^{9}$, a maior freqüência foi de nulíparas $(46,3 \%)$. Os dados da presente pesquisa demonstraram que as nulíparas apresentam maior chance de ter filhos prematuros, mesmo após o tratamento de inibição do TPP, pois tiveram menos sucesso no tratamento quando comparadas com as primíparas. Esse resultado difere de outros trabalhos 14,20 que não constataram diferença significativa entre paridade $\mathrm{e}$ freqüência de parto prematuro.

O despreparo, a insegurança e o medo, em geral, estão presentes nas nulíparas que enfrentam a situação do parto pela primeira vez. O diagnóstico de parto pré-termo, assim como o baixo peso ao nascer geram a ocorrência de estresse psicológico na gravidez. 24

O intervalo interpartal não foi relacionado estatisticamente com o sucesso ou não do tratamento de TPP. Achados similares foram observados por Almeida et al.14 que também não encontraram associação estatística com a prematuridade, estudando o intervalo maior e menor que um ano.

Durante a internação hospitalar da gestante para tratamento de TPP, conclui-se que é essencial observar os aspectos educativos como: repouso, abstinência sexual, identificação precoce das contrações e aconselhar a mulher a procurar imediatamente o hospital no caso de ocorrer qualquer atividade uterina. Essas orientações devem ser dadas à gestante e sua família na alta hospitalar, além disso, poderia também ser implementada a visita domiciliar para acompanhamento dessas mulheres. O envolvimento da família é um fator que poderá contribuir para o sucesso do tratamento do TPP.

\section{Referências}

1. Ministério da Saúde. DATASUS. Sistema de informações sobre nascidos vivos. Disponível em: http://tabnet.datasus. gov.br/cgi/tabcgi.exe?sinasc/cnv/nvuf.def [2006 fev 02].
2. Camargo RPS, Simões JA, Cecatti JG, Alves VMN, Faro S. Impact of treatment for bacterial vaginosis on prematurity among Brazilian pregnant women: a retrospective cohort study. São Paulo Med J. 2005; 123: 108-12. 
3. Cunningham FG, MacDonald PC, Gant NF, Leveno KJ, Gilstrap LC, Hankins GDV, Clark SL. Parto pré-termo. In: Cunningham FG, MacDonald PC, Gant NF, Leveno KJ, Gilstrap LC, Hankins GDV, Clark SL. Williams Obstetrícia. 20. ed. Rio de Janeiro: Guanabara Koogan; 2000. p. 70026.

4. Steer P, Flint C. ABC of labour care: preterm labour and premature rupture of membranes. Br Med J. 1999; 318: 1059-62.

5. Keirse MJNC, Grant A, King JF. Preterm labour. In: Chalmers I, Enkin M, Durse MAN. Effective care in pregnancy and childbirth. Oxford: University Press; 1989. p. 694-745.

6. Keirse MJNC. The history of tocolysis. Br J Obstet Gynaecol. 2003; 110 (Suppl 20): 94-7.

7. Fonseca ESB, Bittar RE, Zugaib M. Prevenção do nascimento prematuro: importância da monitorização das contrações uterinas. Rev Bras Ginecol Obstet. 1999; 21 : 509-15.

8. Lam F, Istwan NB, Rhea D, Smith M, Main E, Slage T, Stanziano G. Evaluation of the pregnancy prolongation index (PPI) as a measure of success of obstetric interventions in the prevention of preterm birth and associated morbidities. Am J Obstet Gynecol. 2005; 192: 2047-54.

9. Spallicci MDB, Chiea MA, Perroni A, Albuquerque PB de, Zugaib M. Uso da isoxsuprina na inibição do trabalho de parto prematuro na Clínica Obstétrica do Hospital Universitário da Universidade de São Paulo. Rev Bras Med. 2000; $57: 65-9$

10. Anotayanonth S, Subhedar NV, Garner P, Neilson JP Harigopal S. Betamimetics for inhibiting preterm labour [review]. Cochrane Database Syst Rev. 2004; (4) [CD004352].

11. Stan C, Boulvain M, Hirsbrunner-Amagbaly P, Pfister R. Hydration for treatment of preterm labour [review]. Cochrane Database Syst Rev. 2005; (2) [CD003096].

12. Collaborative Group on Antenatal Steroid Therapy. Effect of antenatal dexamethasone administration on the prevention of respiratory syndrome. Am J Obstet Gynecol. 1981; 141: $276-96$

13. Shmuel A, Tzipora D, Litmanovitz I, Rivka R, Bauer S, Moshe F. Preterm labour at 34-36 weeks of gestation: should it be arrested? Paediatr Perinat Epidemiol. 2001; 15: 252-6.

Recebido em 16 de março de 2005

Versão final apresentada em 7 de abril de 2006

Aprovado em 26 de abril de 2006
14. Almeida MVL, Silva LGP, Montenegro CAB. A prematuridade na maternidade-escola do Rio de Janeiro: estudo dos fatores de risco. J Bras Ginecol. 1994; 104: 191-7.

15. Zeitlin JA, Saurel-Cubizolles MJ, Ancel PY. Marital status, cohabitation, and the risk of preterm birth in Europe: where births outside marriage are commom and uncommom. Paediatr Perinat Epidemiol. 2002; 16: 124-30.

16. Tsunechiro MA, Bonadio IC. A família na rede de apoio da gestante. Fam Saúde Desenv. 1999; 1: 103-6.

17. Pardo RA, Nazer J, Cifuentes L. Prevalencia al nacimiento de malformaciones congénitas y de menor peso de nacimiento en hijos de madres adolescentes. Rev Med Chil. 2003; 131: 1165-72.

18. Aragäo VMF, Silva AAM, Aragäo LF, Barbieri MA, Bettiol $\mathrm{H}$, Coimbra LC, Ribeiro VS. Risk factors for preterm births in Säo Luís, Maranhäo, Brazil. Cad Saúde Pública. 2004; 20: 57-63.

19. Robaina Castellano GR, Pollo Medina JM, Morales Rigau JM, Robaina Castellanos RE. Análisis multivariada de factores de riesgo de prematuridad em Matanzas. Rev Cubana Obstet Ginecol. 2001; 27: 62-9.

20. Silva LGP, Almeida MVL, Reis AFF, Perreira JL, Carmo $\mathrm{AV}$, Castro KB. Fatores de risco associados à prematuridade: análise multivariada. J Bras Ginecol. 1996; 106: 401-

21. Nascimento LFC. Epidemiolgy of preterm deliveries in Southeast Brazil: a hospital-based study. Rev Bras Saúde Matern Infant. 2001; 1: 263-8.

22. Morais EM, Alflen TL, Spara P, Beitune P. Momento e freqüência das visitas de pré-natal: repercussões sobre os nascimentos pré-termo. Rev Bras Ginecol Obstet. 1998; 20 : 25-32.

23. Ministério da Saúde. Secretaria de Políticas. Assistência pré-natal: manual técnico. Brasília (DF); 2000.

24. Rondó PH, Ferreira RF, Nogueira F, Ribeiro MC, Lobert H, Antes R. Maternal psychological stress and distress predictors of low birth weight, prematurity and intrauterine growth retardation. Eur J Clin Nutr. 2003; 57: 266-72. 